Musées, Patrimoine et Culture scientifiques et techniques

$126 \mid 2009$

novembre - décembre 2009

\title{
L'observatoire mis en place par le Cirasti, mouvement français des Exposciences
}

Testimonials of evaluation practices

Olivier Las Vergnas

\section{OpenEdition}

\section{Journals}

Édition électronique

URL : http://journals.openedition.org/ocim/216

DOI : 10.4000/ocim. 216

ISSN : 2108-646X

Éditeur

OCIM

Édition imprimée

Date de publication : 1 novembre 2009

Pagination : 44-48

ISSN : 0994-1908

Référence électronique

Olivier Las Vergnas, «L'observatoire mis en place par le Cirasti, mouvement français des

Exposciences », La Lettre de I'OCIM [En ligne], 126 | 2009, mis en ligne le 01 novembre 2011, consulté le 22 avril 2019. URL : http://journals.openedition.org/ocim/216 ; DOI : 10.4000/ocim.216

Tous droits réservés 


\title{
L'observatoire mis en place par le Cirasti, mouvement français des Exposciences
}

\author{
Olivier Las Vergnas *
}

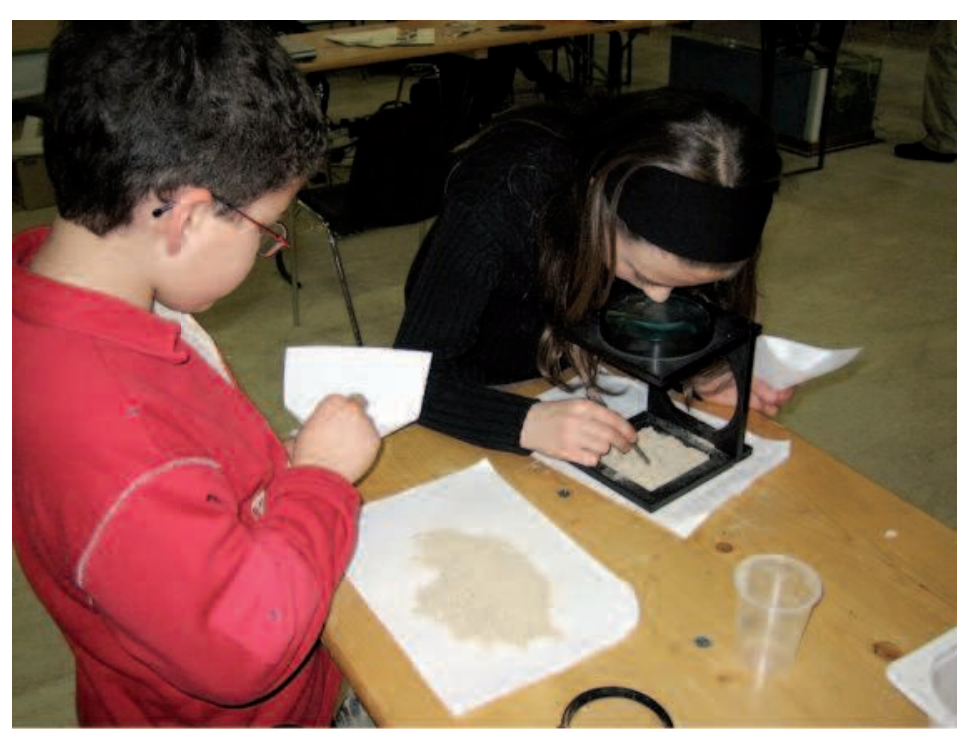

Expérimentation devant le public lors d'une Exposcience (C) DR/ Cirasti

* Olivier Las Vergnas est vice-président du Cirasti, mouvement français des Exposciences olivier.lasvergnas@cite-sciences.fr

\section{Les Exposciences :}

un cercle vertueux pour favoriser

les projets de découverte scientifique

Les activités de découverte scientifique passionnent tous les jeunes à condition de créer les conditions d'émergence et d'expression de cette passion : appropriation, progression, échanges, aboutissement, valorisation. Le développement de projets, pourvu qu'ils permettent des concrétisations gratifiantes, est un des meilleurs moyens de l'appropriation de savoirs. De tels projets offrent aussi l'opportunité de développer, à partir de résolutions de problèmes et de situations de communication, l'estime de soi, l'esprit critique et de multiples savoir être.

Partant de ce constat, le Cirasti, mouvement français des Exposciences (http://cirasti.org), a été créé voici 24 ans par les mouvements nationaux d'éducation populaire motivés par le développement des activités de découverte scientifique : d'une part des mouvements généralistes, comme les Ceméa, les deux fédérations nationales de MJC, les Francas, les Éclaireurs de France, la Ligue de l'enseignement, la fédération Léo Lagrange, les Foyers ruraux et d'autre part des associations spécialisées, comme Planète Sciences, Les Petits Débrouillards, l'association française d'Astronomie.

Concrètement, le Cirasti développe les « Exposciences » où des centaines de jeunes présentent pendant quelques jours plusieurs dizaines de projets. Ce sont des collectifs territoriaux qui, dans vingt régions et dans plus de vingt départements, les organisent : ils réunissent au total plus de trois cents représentants d'associations régionales, départementales et locales, de structures éducatives, de l'Éducation nationale, des services déconcentrés de l'État 
et de collectivités territoriales en lien avec des CCSTI, des laboratoires de recherche et des entreprises. Depuis 1985, date de la création du Cirasti, 463 Exposciences régionales ou départementales ont été organisées, dans lesquelles 9390 équipes de jeunes ont présenté leurs projets scientifiques ou techniques. 450 projets de jeunes français ont par ce biais participé ensuite à l'une des 14 Exposciences internationales coordonnées par le Milset (Mouvement international pour le loisir scientifique et technique qui s'est développé sur 54 pays), sans oublier les 78 équipes qui ont présenté leurs projets lors de 7 Exposciences européennes.

Ce concept d'Exposciences est formalisé par une charte qui en précise les principes et les conditions de réussite, mais il fait aussi l'objet d'adaptations pour plus de proximité ou d'accessibilité. Ainsi depuis 1994, 602 projets de jeunes ont été présentés lors de 154 manifestations locales ou cantonales intitulées «Sciences Buissonnières » d'une ampleur plus limitée, mais plus souples à organiser que les manifestations régionales ou départementales.

De fait, ces Exposciences constituent un maillon clef d'un cercle vertueux qui favorise à la fois l'accomplissement et la démultiplication de ce type de projets : d'une part elles offrent aux équipes qui les développent un jalon important dans leur travail, leur permettant de mieux le structurer, d'en mieux gérer les échéances et surtout de préparer sa communication, d'autre part elles donnent à voir à de multiples publics (autres jeunes, parents, animateurs, relais d'opinion) l'intérêt et l'opportunité que peuvent représenter de tels projets de découverte scientifique.

\section{Les Exposciences comme analyseur des pratiques scientifiques}

Les Exposciences constituent aussi un excellent analyseur des activités scientifiques et techniques des jeunes; de ce constat est née l'idée de créer au sein du Cirasti un observatoire des projets qui y sont présentés. Les critères étudiés concernent des critères qui nous préoccupent spécifiquement, à savoir la nature des projets dans leur relation aux savoirs (expérimentation, présentation documentaire, dispositif technique), leur cadre de réalisation (ratios entre les projets à support scolaire, périscolaire ou entièrement extrascolaire) et leur thématique (discipline, ou passerelle vers l'approche scientifique). Sur ce dernier point, en effet, nous entendons particulièrement impulser le développement de projets d'inspiration technique, artistique ou vie quotidienne, projets qui permettent d'accueillir et de dynamiser des jeunes, projets plus diversifiés que ceux directement ancrés sur des disciplines et corpus scolaires. Ce qui nous intéresse en la matière étant bien entendu de favoriser des projets correspondant à de réels centres d'intérêt ou de préoccupations des jeunes eux-mêmes, le moins possible téléguidés par des adultes. Dans le cadre du Milset (http://milset.org) il est prévu un développement international permettant ainsi des comparaisons sur les 54 pays organisant des Exposciences de jeunes au sein de ce mouvement.

À terme, l'observatoire des Exposciences devrait dépasser ce premier niveau de description des projets et s'intéresser également à la diversification du public touché : pourraient ainsi être observées la multiplication des projets d'une année sur l'autre et surtout la capacité à dépasser la pure consanguinité qui caractériserait une Exposcience visitée uniquement par les proches des jeunes exposants. Afin d'estimer la représentativité de chaque Exposcience, nous voudrions aussi nous faire une idée du réservoir de projets potentiels sur un territoire que l'on pourrait voir à terme converger pour renforcer les manifestations existantes, mais la méthode d'évaluation reste là à inventer. À noter que derrière cette question se cache un champ d'investigation crucial pour ceux qui s'intéressent à objectiver la question de l'intérêt pour les sciences : celui de l'évolution du « loisir scientifique », qui existe de moins en moins en France hors du champ périscolaire, sauf dans quelques domaines très spécialisés des sciences amateurs, comme l'astronomie.

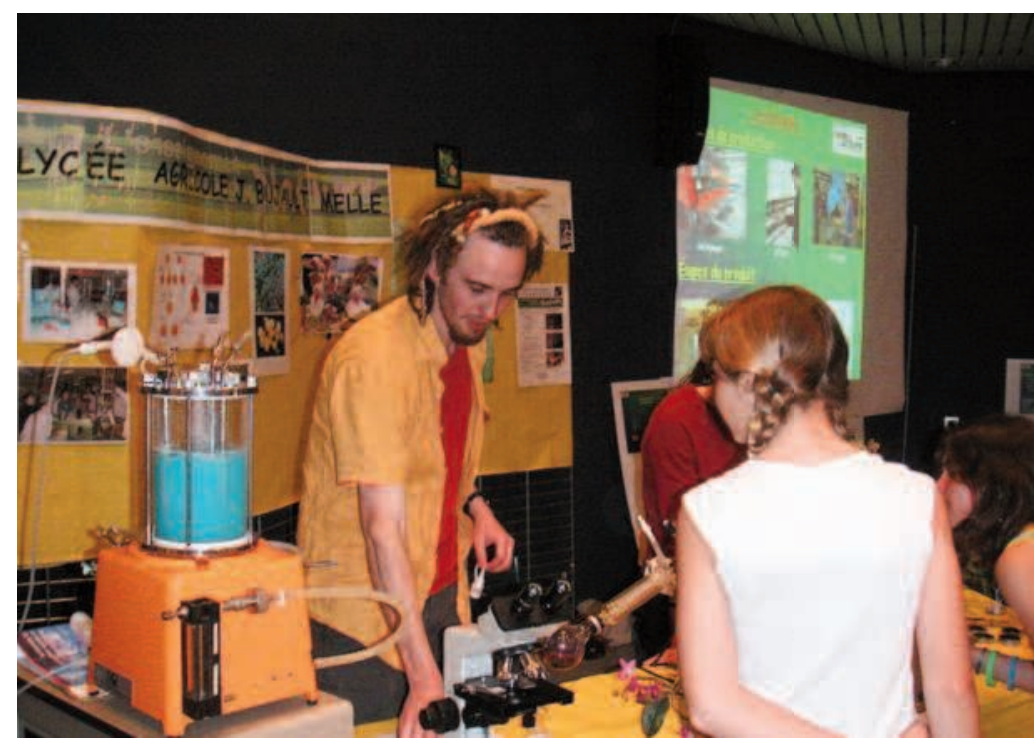

Exposcience du Cirasti en Poitou-Charente (c) DR/Cirasti 


\section{Quelques exemples de résulitits à propos des Exposciences 2008}

La part des associations dans les projets d'Exposciences On observe toujours (relativement aux années précédentes et dans une proportion comparable) une majorité de projets d'origine scolaire parmi ceux valorisés dans les Exposciences 2008. Notre présentation prend en compte non la distinction stricte entre projets d'origine scolaire et hors scolaire stricto sensu, mais celle entre les projets purement scolaires d'une part, et ceux où une association est impliquée : soit qu'elle les ait portés seule, soit qu'elle y ait contribué au titre de partenaire ou d'intervenant (éventuellement à sa propre initiative). Dans les barres ci-dessous, la partie bleue représente les projets purement scolaires, et la partie orangée les projets où une association est impliquée selon l'un des modes ci-dessus. La proportion est de deux tiers pour un tiers. Si l'on ramène cette proportion au nombre de jeunes touchés respectivement par l'école et les structures associatives, ainsi qu'au temps qu'ils y passent, nous ne pouvons que conclure que dans ce paysage, l'éducation populaire est surreprésentée et que son impact est proportionnellement très considérable. Au-delà de la moyenne nationale, les disparités entre Exposciences régionales restent très fortes, et relèvent de l'histoire de chaque collectif régional.

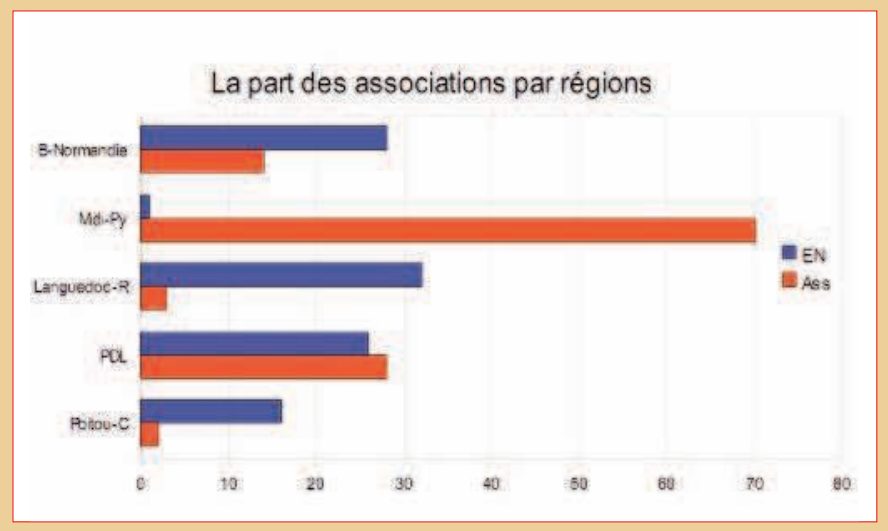

Répartition des projets par origines lécoles, lycées, collèges, structures de loisirs)

On voit dans cette étude des tendances par régions (que ce soit sur l'axe des âges ou celui des structures d'origine) ; majorité de projets lycéens en Languedoc-Roussillon, d'écoles élémentaires en Midi-Pyrénées et Basse-Normandie, près de $50 \%$ de projets associatifs en Pays de la Loire...
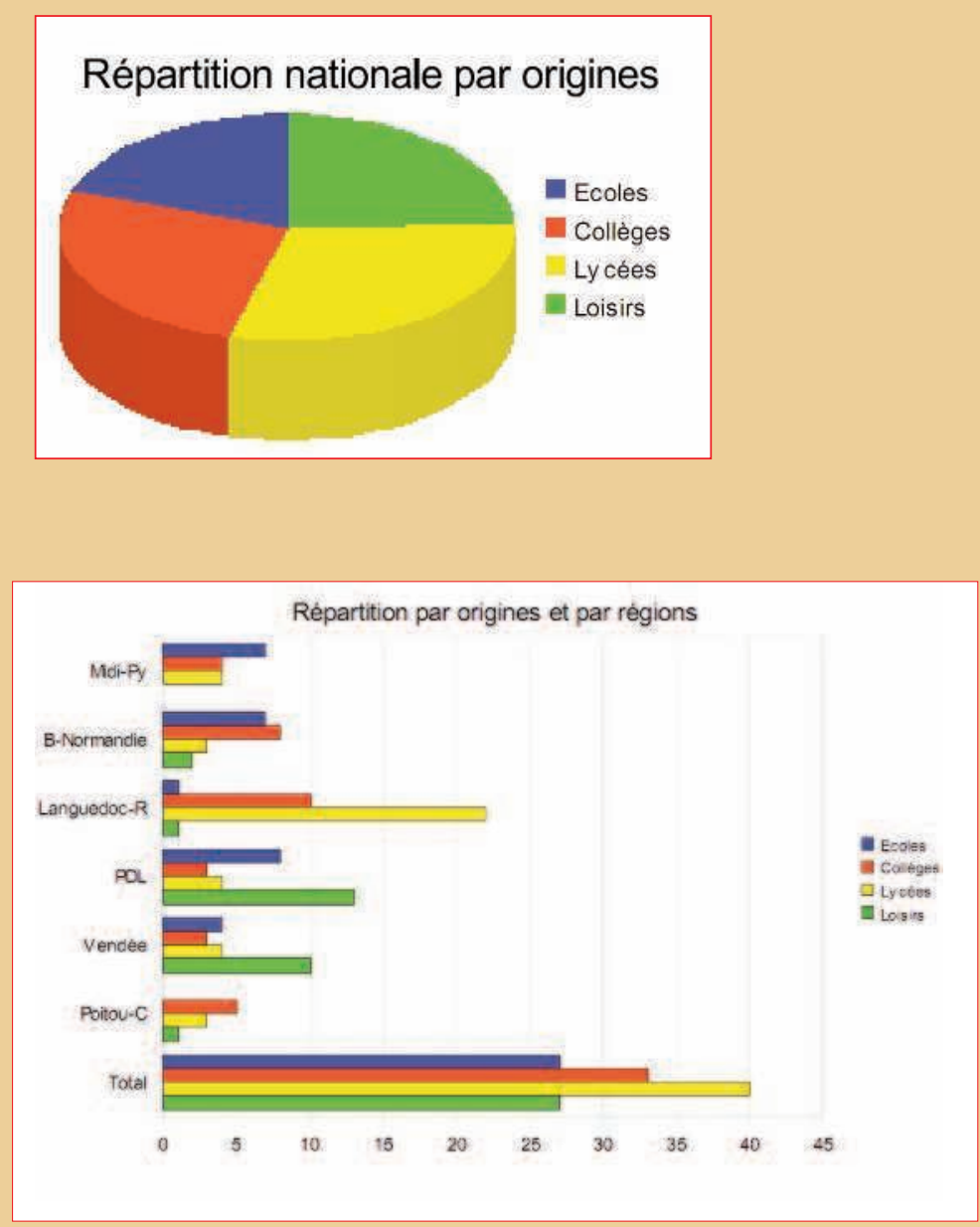

Répartition des participants aux Exposciences 2008 entre filles et garçons

Dans l'ensemble, on note une légère disparité entre filles et garçons, ce qui n'est pas étonnant du point de vue sociologique s'agissant des activités scientifiques et techniques. Cependant il faut noter que lorsque des classes entières réalisent un projet, c'est naturellement à parité, ce qui signifie que les ateliers scientifiques, les IDD et TPE, les clubs de loisirs, donc tous les cadres où les publics ne sont pas captifs, connaissent une moindre implication des filles. Une étude plus fine permettrait de nuancer cette disparité selon les thématiques. Par régions, les différences sont fortes entre la quasi parité des Pays de Loire et le 2/3-1/3 de Poitou-Charente. Un lien statistique serait à faire entre ce rapport et les origines des projets : la prépondérance des jeunes d'âge primaire a peut-être une importance. 


\section{par Joël Le Bras, délégué général du Cirasti de 2002 à 2008}

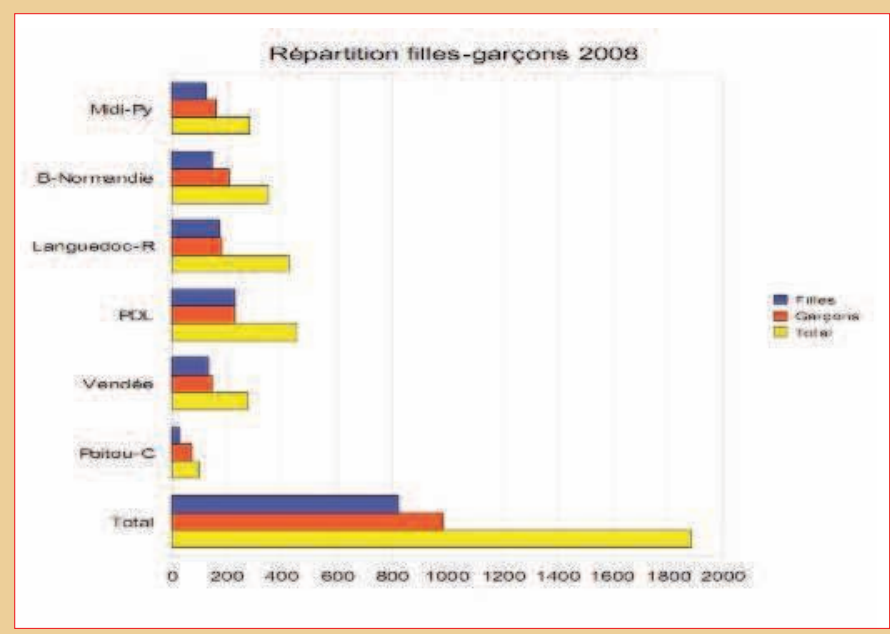

Répartition des projets par thématiques

Nomenclature des thématiques : EEDD (Éducation à l'Environnement et/ou Développement Durable), Bio (Biologie, Sciences de la Vie), Météo-Clim (Sciences du temps et du climat, y compris ballons stratosphériques), Sc-Phys (Sciences expérimentales, Physique), Technique (projets dédiés à la construction d'objets techniques), ST (Sciences de la Terre), Astro-espace (Astronomie et disciplines liées à la conquête spatiale).

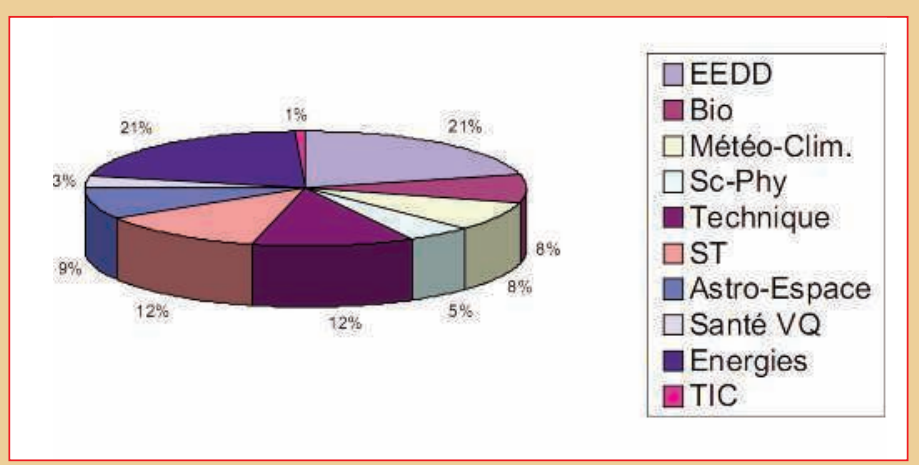

On voit une prédominance relative mais nette, des projets liés à l'environnement, aux énergies, au développement durable, au climat, et aux sciences de la vie : la moitié du total des projets présentés en France dans les Exposciences 2008. Les projets voués aux TIC sont ici peu nombreux, mais on n'a pris en compte que ceux dont la technologie de la communication était le sujet propre ; beaucoup d'autres projets utilisent les TIC pour leur présentation, la recherche documentaire...
Répartition des projets par type de démarche

La démarche expérimentale consiste à répondre par l'expérimentation à une question initiale ; la démarche documentaire consiste à étudier les connaissances dans un domaine et à en faire l'état ; la démarche technique consiste à concevoir et fabriquer un objet technique répondant à un cahier des charges. La plupart des projets Exposciences utilisent plus d'une démarche, et un nombre significatif utilise les trois au cours de la réalisation. Il est notable que la démarche expérimentale est soit la seule mise en œuvre, soit au moins présente, dans les trois quarts des projets. Ce constat pouvait déjà être fait lors de l'étude précédente, ce qui montre l'attachement de l'ensemble des éducateurs à cette démarche. Une étude plus fine ne montre pas d'écart significatif sur ce plan entre les structures de loisirs et l'Éducation nationale. Cependant, si l'on considère les tranches d'âges, il apparaît que les activités lycéennes sont plus axées sur une seule démarche que celles des autres tranches d'âge.

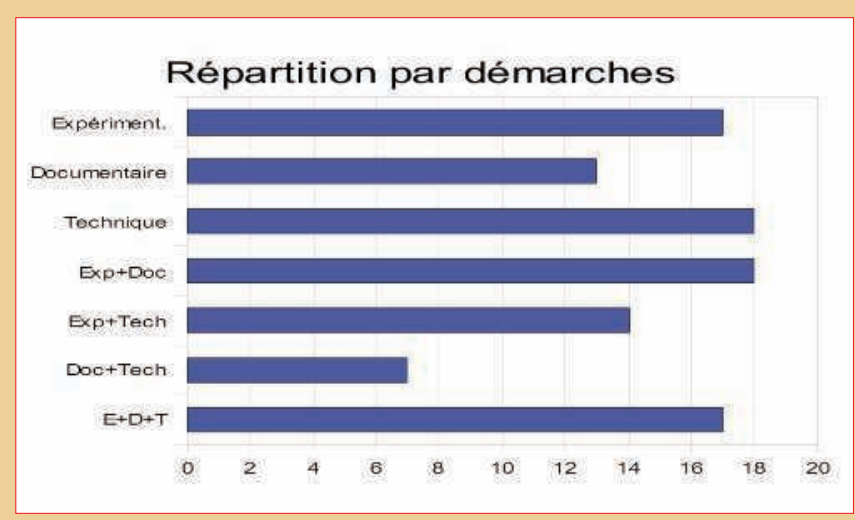

Statistique globale

Les projets présentés par le mouvement français des Exposciences en 2008 ont représenté un total de 260000 heures d'activités scientifiques, à raison d'une moyenne de 15 jeunes et de 50 heures d'activité par projet. Sur l'ensemble du cycle de deux années d'Exposciences régionales, le total d'heures d'activités dépasse largement 500 000, auxquelles il faut ajouter le temps de présence dans les Exposciences, en moyenne 20 heures pour 5000 jeunes, soit 100000 heures supplémentaires. 


\section{L'observatoire, pour une réflexion plus générale entre évaluation choisie et évaluation subie}

Le Cirasti a décidé de mettre en place son observatoire afin de mieux visualiser son rôle et son impact et de permettre aux réseaux impliqués dans les Exposciences de prendre l'initiative en matière d'évaluation de leurs actions. Mais, vu l'évolution générale des relations entre les pouvoirs publics et les mouvements d'éducation populaire ou d'action culturelle, cette question se révèle ne plus être seulement une affaire pédagogique interne. En effet, comme l'a rappelé la troisième édition des rencontres du Cirasti, coorganisées les 31 janvier et 1 er février 2008 avec l'IUT de Tours sur le thème de l'animation scientifique ou technique et l'éducation à l'environnement face au marché, l'époque n'est plus celle des subventions sur des objectifs d'intérêt général, mais celle de la vente de prestations formatées.

En conséquence, avoir développé ses propres critères ou indicateurs d'évaluation, savoir reformuler leurs objectifs et finalités éducatives en résultats évaluables est indispensable aux réseaux éducatifs. Sans cela, ils se retrouveront face à des commissions des marchés en manque de critères pertinents d'efficacité, réduites à juger en seul terme de coût brut. Rien de plus dangereux, alors que certains opérateurs peu scrupuleux, commencent à donner priorité aux activités saucissonnées, aux thématiques racoleuses, mélangeant à l'occasion le scientifique et l'ésotérique.

Dans cette nécessité de disposer d'outils d'observation et de mesure partageables avec les commanditaires, des observatoires comme celui du Cirasti seront précieux. La meilleure stratégie pour ne pas subir des évaluations arbitraires est en effet d'avoir élaboré et fait partager des indicateurs pour donner l'opportunité aux commanditaires de prendre en compte la pertinence et l'efficacité des actions et pas seulement le moindre coût. On ne peut que souhaiter que nos observatoires servent de cadre à une telle concertation sur des critères partagés d'évaluation à proposer aux partenaires et maîtres d'ouvrage.

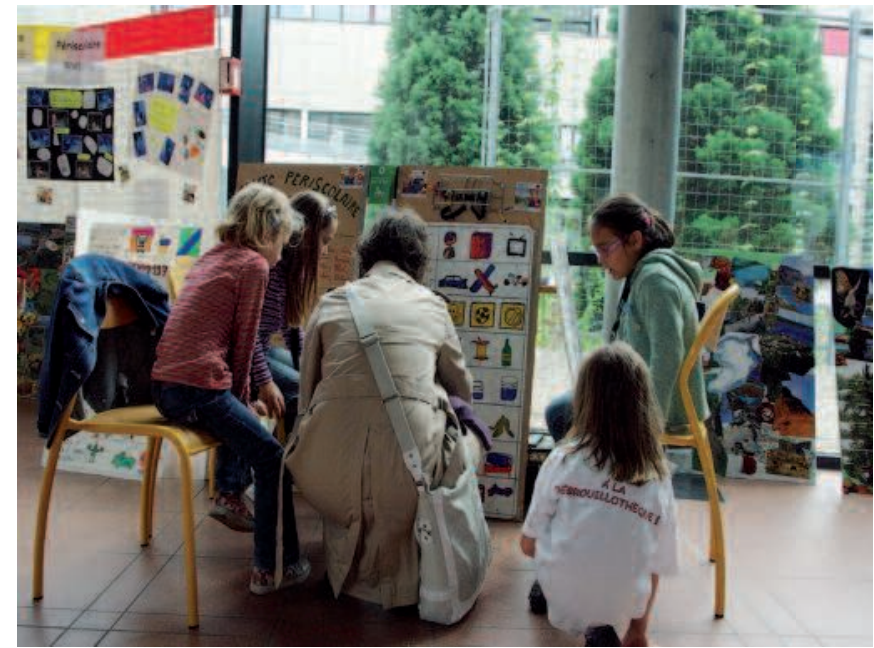

Un groupe «petits débrouillards » à Exposcience Alsace (C) DR/Cirasti

Mais, au-delà de cet angle technocratique, nos observatoires ont aussi une responsabilité sociale, plus globale : celle d'aider à rendre les discours plus précis et concrets en matière de vision de la fameuse « culture scientifique ». Après plusieurs décennies passées à en rappeler chroniquement la nécessité pour tous, on entend encore ici et là des prises de parole qui amalgament la question de l'intérêt pour les sciences du plus grand nombre et la présumée « désaffection des études scientifiques » confondant de fait la question de la détection de l'élite avec celle de la réduction des inégalités vis-à-vis des savoirs. Vivement que nos observatoires fournissent des tableaux de bord permettant de doter nos hommes politiques d'objectifs observables et de distinguer à coup sûr entre pures déclarations d'intention et réelles politiques efficientes.

\section{Bibliographie}

\footnotetext{
Las Vergnas, O. et Vannier, P. Les Exposciences : pourvu que l'on s'en donne les moyens, tous les jeunes se passionnent pour la découverte scientifique, Revue du Centre de Recherche et d'information sur la littérature de Jeunesse, n87, 2006.
}

Las Vergnas, O. Les savoirs scientifiques seront-ils toujours infantilisants ?, Alliage, culture, science, technique. n59, 2006. 